Check for updates

Cite this: Phys. Chem. Chem. Phys., $2019,21,23111$

Received 22nd May 2019,

Accepted 28th August 2019

DOI: $10.1039 / c 9 c p 02907 a$

rsc.li/pccp

\section{Evaluating lithium diffusion mechanisms in the complex spinel $\mathrm{Li}_{2} \mathrm{NiGe}_{3} \mathrm{O}_{8} \dagger$}

\author{
Daniel Z. C. Martin, (D) a Abby R. Haworth, (D) ${ }^{b}$ Whitney L. Schmidt, ${ }^{c}$ \\ Peter J. Baker, (D) ${ }^{d}$ Rebecca Boston, (D) ${ }^{a}$ Karen E. Johnston (D) ${ }^{b}$ and \\ Nik Reeves-McLaren (D) *a
}

\begin{abstract}
Lithium-ion diffusion mechanisms in the complex spinel $\mathrm{Li}_{2} \mathrm{NiGe}_{3} \mathrm{O}_{8}$ have been investigated using solidstate NMR, impedance, and muon spectroscopies. Partial occupancy of migratory interstitial $12 d$ sites is shown to occur at lower temperatures than previously reported. Bulk activation energies for $\mathrm{Li}^{+}$ion hopping range from $0.43 \pm 0.03 \mathrm{eV}$ for powdered samples to $0.53 \pm 0.01 \mathrm{eV}$ for samples sintered at $950{ }^{\circ} \mathrm{C}$ for $24 \mathrm{~h}$, due to the loss of $\mathrm{Li}$ during sintering at elevated temperatures. A lithium diffusion coefficient of $3.89 \times 10^{-12} \mathrm{~cm}^{2} \mathrm{~s}^{-1}$ was calculated from muon spectroscopy data for $\mathrm{Li}_{2} \mathrm{NiGe}_{3} \mathrm{O}_{8}$ at $300 \mathrm{~K}$.
\end{abstract}

\section{Introduction}

Since Sony's successful commercialisation of lithium-ion batteries (LIBs) in 1990, their numerous desirable properties have been widely reported. ${ }^{1-3}$ However, despite becoming ubiquitous in modern society, several safety concerns still remain, predominantly related to the use of flammable and corrosive organic liquid electrolytes in close proximity to highly energetic electrode materials. ${ }^{4-6}$ Whilst accidents are relatively rare, these liquid electrolytes can pose significant dangers to consumers and other end-users, as well as reputational damage for device manufacturers. ${ }^{7}$

An all-solid-state battery utilising a solid electrolyte could alleviate many of these concerns, whilst offering improved shock resistance and durability. ${ }^{8}$ To date, research has primarily focused on the development and optimisation of materials with room-temperature $\mathrm{Li}^{+}$ion conductivities $\left(\sigma_{\mathrm{i}}\right)$ of $\geq 10^{-3} \mathrm{~S} \mathrm{~cm}^{-1}$, in order to compete with current commercial liquid electrolytes. ${ }^{8}$ Several such materials exist, for instance thio-LISICONs (e.g., $\mathrm{Li}_{10} \mathrm{GeP}_{2} \mathrm{~S}_{12}, \sigma_{\mathrm{i}}=1.2 \times 10^{-2} \mathrm{~S} \mathrm{~cm}^{-1}$ ) and Li-stuffed garnets (e.g., $\left.\mathrm{Li}_{6.4} \mathrm{La}_{3} \mathrm{Zr}_{1.4} \mathrm{Ta}_{0.6} \mathrm{O}_{12}, \sigma_{\mathrm{i}}=10^{-3} \mathrm{~S} \mathrm{~cm}^{-1}\right){ }^{9-11}$ Nevertheless, many of these candidate materials suffer a number of drawbacks, which are preventing their commercialisation. For example,

\footnotetext{
${ }^{a}$ Department of Materials Science and Engineering, University of Sheffield, Sheffield, S13JD, UK. E-mail: n.reeves@sheffield.ac.uk

${ }^{b}$ Department of Chemistry, Durham University, Durham, DH1 3LE, UK. E-mail: karen.johnston@durham.ac.uk

${ }^{c}$ Division of Natural Sciences and Mathematics, Kentucky Wesleyan College, 3000 Frederica St., Owensboro, KY 42301, USA

${ }^{d}$ ISIS Facility, Science and Technology Facilities Council, Rutherford Appleton Laboratory, Harwell Science and Innovation Campus, Didcot, Oxfordshire, OX11 OQX, UK

$\dagger$ Electronic supplementary information (ESI) available. See DOI: 10.1039/c9cp02907a
}

thio-LISICONs are unstable in air and decompose at cell voltages $>4 \mathrm{~V} v$ s. $\mathrm{Li} / \mathrm{Li}^{+}{ }^{12,13}$ Many are also reported to react with lithium metal. ${ }^{12}$ Li-stuffed garnets can undergo proton exchange $\left(\mathrm{H}^{+} / \mathrm{Li}^{+}\right)$, substantially reducing levels of $\mathrm{Li}^{+}$ion conductivity. ${ }^{14,15}$ They are also widely reported to exhibit high interfacial resistance with electrode materials (up to $\sim 2000 \Omega \mathrm{cm}^{2}$ ). ${ }^{16}$ One solution to lowering this interfacial resistance is to use materials with the same crystal structure to enable lattice matching, for example by developing an all-spinel solid-state battery.

The spinel structure, $\mathrm{AB}_{2} \mathrm{O}_{4}$, has been studied extensively and is known to consist of cubic close packed $\mathrm{O}^{2-}$ anions, with A-site cations occupying an eighth of the tetrahedral sites and B-site cations filling half of the available octahedral sites for 'normal' spinels. ${ }^{17,18}$ Depending on the cations present, the spinel structure may also be inverse $\left([\mathrm{B}]^{\text {tet }}[\mathrm{A}, \mathrm{B}]^{\text {oct }} \mathrm{O}_{4}\right)$ or random $\left(\left[\mathrm{B}_{0.67} \mathrm{~A}_{0.33}\right]^{\text {tet }}\left[\mathrm{A}_{0.67} \mathrm{~B}_{1.33}\right]^{\text {oct }} \mathrm{O}_{4}\right)$. Cubic spinels generally crystallise in the $F d \overline{3} m$ space group. In Li-based spinels, $\mathrm{Li}^{+}$ion conductivity typically follows a two-step conduction mechanism involving $\mathrm{Li}$ ions in $8 a$ tetrahedral sites hopping into empty $16 c$ octahedral sites and then onto adjacent $8 a$ sites, forming a three-dimensional $8 a-16 c-8 a$ pathway for ionic migration ${ }^{19}$ that means spinels often exhibit interesting and useful electrical properties. As a result, several manganese-based spinels have been considered as potential high voltage cathode materials $\left(>4.7 \mathrm{~V} v\right.$ s. $\left.\mathrm{Li}^{+} / \mathrm{Li}\right)$, e.g., $\mathrm{LiCoMnO}_{4}$ and $\mathrm{LiNi}_{0.5} \mathrm{Mn}_{1.5} \mathrm{O}_{4}{ }^{20-22}$ However, these are incompatible with current LIB cell designs due to the breakdown of organic electrolytes above ca. $4.5 \mathrm{~V}$ vs. $\mathrm{Li}^{+} / \mathrm{Li}$. Spinel-based titanates such as $\mathrm{Li}_{4} \mathrm{Ti}_{5} \mathrm{O}_{12}$ are currently of interest as anode materials in LIB applications, as they exhibit excellent dimensional stability during Li (de)intercalation. ${ }^{23,24}$ In contrast, spinel-based materials for solid electrolyte applications have received considerably less attention, since they often incorporate redox active transition metals. One potential candidate material is $\mathrm{Li}_{2} \mathrm{NiGe}_{3} \mathrm{O}_{8}$, previously 
reported by Kawai et al. to exhibit a modest $\mathrm{Li}^{+}$ion conductivity of $\sim 10^{-7} \mathrm{~S} \mathrm{~cm}^{-1}$ at $63{ }^{\circ} \mathrm{C}$ (comparable to the tetragonal phase of the Li-stuffed garnet $\mathrm{Li}_{7} \mathrm{La}_{3} \mathrm{Zr}_{2} \mathrm{O}_{12}$ ) and which is electrochemically inactive below $5 \mathrm{~V} v s . \mathrm{Li}^{+} / \mathrm{Li}^{25}$

$\mathrm{Li}_{2} \mathrm{NiGe}_{3} \mathrm{O}_{8}$ can be described as an ordered or 'complex' spinel in space group $\mathrm{P}_{3} 32$, with $\mathrm{Li}^{+}$ions occupying tetrahedral $8 c$ sites, and $1: 3$ cation ordering of $\mathrm{Ni}$ and Ge on the $4 b$ and $12 d$ octahedral sites, respectively. ${ }^{25}$ Conduction pathways in complex spinels are less well studied compared to normal spinels, but can involve additional steps depending on the nature of the ordering present. Previous work using variable-temperature time-of-flight neutron powder diffraction (ToF ND) indicated the presence of a second $\mathrm{Li} 12 d$ octahedral site in $\mathrm{Li}_{2} \mathrm{NiGe}_{3} \mathrm{O}_{8}$ at high temperatures $\left(\sim 350-850{ }^{\circ} \mathrm{C}\right.$ ), leading to the proposition of a three-dimensional $8 c-12 d-8 c$ conduction pathway. ${ }^{26}$ Theoretical studies by Nakayama et al. predicted the activation energy for this particular pathway to be $0.47 \mathrm{eV}$, lower than other previously proposed pathways. ${ }^{19}$

An improved understanding of ionic diffusion mechanisms in spinel materials, and $\mathrm{Li}^{+}$ion conductors at large, is crucial if such materials are to be improved and optimised for use as solid electrolytes in all-solid-state LIBs. To this end, we have used a combination of techniques, including impedance, muon and solid-state NMR spectroscopies to enhance our understanding of the crystal structure of the complex spinel $\mathrm{Li}_{2} \mathrm{Ni}$ $\mathrm{Ge}_{3} \mathrm{O}_{8}$ and the $\mathrm{Li}^{+}$ion diffusion dynamics therein.

\section{Experimental methods}

\subsection{Synthesis and characterisation}

Samples of $\mathrm{Li}_{2} \mathrm{NiGe}_{3} \mathrm{O}_{8}$ were synthesised via traditional solid state methods. Stoichiometric amounts of $\mathrm{Li}_{2} \mathrm{CO}_{3}$ (Sigma-Aldrich, 99\%), NiO (Alfa Aesar, 99\%) and $\mathrm{GeO}_{2}$ (Sigma-Aldrich, 99.999\%) were mixed and ground in an agate mortar and pestle, placed into $\mathrm{Au}$ boats and fired at $923 \mathrm{~K}$ for 5 hours. Prior to heating, the starting reagents were dried at either 453 (for $\mathrm{Li}_{2} \mathrm{CO}_{3}$ ) or $773 \mathrm{~K}$ (for oxides) for 12 hours. The samples were then calcined at $1173 \mathrm{~K}$ for 36 hours, with intermediate regrinding. Identical specimens of $\mathrm{Li}_{2} \mathrm{NiGe}_{3} \mathrm{O}_{8}$ were used for all XRD, impedance, solid-state NMR and $\mu$ SR studies.

Samples were characterised by X-ray diffraction (XRD) using a Bruker D2 Phaser diffractometer with $\mathrm{Cu} \mathrm{K} \alpha(\lambda=1.5418 \AA$ A $)$ radiation. The ICDD's PDF-4+ database (2019 edition) and SIeve+ software were used for phase analysis. Rietveld refinements were performed using the GSAS-II software. ${ }^{27}$

\subsection{Impedance spectroscopy}

Pellets of $\mathrm{Li}_{2} \mathrm{NiGe}_{3} \mathrm{O}_{8}$ were prepared for impedance spectroscopy measurements by uniaxial pressing. Pellets were placed onto sacrificial powder in an alumina crucible and sintered at $1223 \mathrm{~K}$ for 6 or 24 hours, resulting in pellets with $66 \%$ and $81 \%$ density, respectively. Both pellet faces were polished and gold electrodes applied to each as a paste. Each pellet was then annealed at $1123 \mathrm{~K}$ for 2 hours. All heating steps described used heating/cooling rates of $5{ }^{\circ} \mathrm{C} \mathrm{min}^{-1}$. Impedance measurements were collected using a Solartron Analytical/Ametek ModuLab XM MTS system from $1 \mathrm{MHz}$ to $30 \mathrm{mHz}$ with a $100 \mathrm{mV}$ applied AC voltage. Measurements were taken during heating from 336 to $541 \mathrm{~K}$.

\subsection{Solid-state NMR spectroscopy}

Solid-state NMR spectra were acquired using a Bruker 500 Avance III HD spectrometer, equipped with a wide-bore $11.7 \mathrm{~T}$ Oxford magnet, using Larmor frequencies of $194.4 \mathrm{MHz}$ for ${ }^{7} \mathrm{Li}(I=3 / 2)$ and 73.6 $\mathrm{MHz}$ for ${ }^{6} \mathrm{Li}(I=1)$. Powdered samples were packed into conventional 4.0 or $1.3 \mathrm{~mm} \mathrm{ZrO}_{2}$ rotors and magic-angle spinning (MAS) rates of 10 and $60 \mathrm{kHz}$ were employed, respectively. Both ${ }^{6} \mathrm{Li}$ and ${ }^{7} \mathrm{Li}$ chemical shifts were referenced to $1 \mathrm{M} \mathrm{LiCl}_{(\mathrm{aq})}$.

Conventional ${ }^{7}$ Li MAS NMR spectra were obtained using a single-pulse experiment with typical pulse lengths of $3 \mu \mathrm{s} .{ }^{6} \mathrm{Li}$ MAS NMR spectra were acquired using a Hahn echo experiment $\left(90_{x}-\tau-180_{y}\right)$ with a typical $\pi / 2$ pulse length of $3 \mu$ s. Typical radiofrequency field strengths of $84 \mathrm{kHz}$ were employed and an experimentally optimised recycle interval of $0.2 \mathrm{~s}$ was used for both ${ }^{7} \mathrm{Li}$ and ${ }^{6} \mathrm{Li}$. Standard variable-temperature (VT) ${ }^{6} \mathrm{Li}$ MAS NMR experiments were completed between 260 and $412 \mathrm{~K}$ using conventional hardware and the parameters stated above. In all cases, temperatures were calibrated using the ${ }^{1} \mathrm{H}$ signal of $\mathrm{MeOH}$; quoted temperatures thus reflect the true sample temperature during the experiment.

Additional static variable-temperature ${ }^{6} \mathrm{Li}$ NMR experiments were completed for $\mathrm{Li}_{2} \mathrm{NiGe}_{3} \mathrm{O}_{8}$ over the temperature range 204 to $609 \mathrm{~K}$ using a Bruker 400 Avance III HD spectrometer, equipped with a wide-bore $9.7 \mathrm{~T}$ magnet, using a Larmor frequency of $58.9 \mathrm{MHz}$ for ${ }^{6} \mathrm{Li}$. The sample was packed into a $5.0 \mathrm{~mm}$ glass tube and placed into a Bruker $5.0 \mathrm{~mm}$ static probe. The static NMR spectra were acquired using a standard solid echo experiment $\left(90_{x}-\tau-90_{y}\right)$, with a typical $\pi / 2$ pulse length of $5 \mu \mathrm{s}$. Here, $\tau$ represents an echo delay of $200 \mu \mathrm{s}$ between 197 and $542 \mathrm{~K}$ and $250 \mu \mathrm{s}$ at $609 \mathrm{~K}$. The interpulse delay was increased in order to acquire the whole echo at higher temperatures. Typical radiofrequency field strengths of $50 \mathrm{kHz}$ were employed and the experimentally optimised recycle interval was $0.05 \mathrm{~s}$. In all cases, true sample temperatures during experiments have been determined using a lead nitrate calibrant.

\subsection{Muon spin relaxation ( $\mu \mathrm{SR})$ spectroscopy}

All $\mu$ SR measurements were performed using the EMU instrument at the ISIS pulsed muon facility. Around $\sim 1 \mathrm{~g}$ of sample was packed into a disk of $30 \mathrm{~mm}$ diameter and $1.5 \mathrm{~mm}$ thickness and sealed in a Ti sample holder with a $\sim 25 \mu \mathrm{m}$ thick Ti foil window. The sample was implanted with $3.2 \mathrm{MeV}$ spin-polarised positive muons, and the out-coming positrons detected by 96 scintillator segments arranged in two circular arrays. Data were collected from 39-555 K using a closed cycle refrigerator under three different applied longitudinal magnetic fields $(0,5$ and $10 \mathrm{G})$. Initial symmetry calibrations were conducted using a $20 \mathrm{G}$ transverse magnetic field. Data analysis was conducted using the WiMDA program. $^{28}$ Collection of data in multiple longitudinal fields enabled (i) the controlled decoupling of interactions between the implanted muons and any local magnetic field distributions, and (ii) additional constraints in and hence more reliable results from the fitting process. 


\section{Results and discussion}

Room-temperature powder XRD data for $\mathrm{Li}_{2} \mathrm{NiGe}_{3} \mathrm{O}_{8}$ are shown in Fig. 1. Bragg peaks were indexed to the $P 4_{3} 32$ space group, with no additional or missing reflections, giving a refined lattice parameter $a=8.1848(5) \AA$, in good agreement with previous reports. $^{25,26}$ Details of the corresponding Rietveld refinement and a list of refined structural parameters are given in the ESI. $\dagger \mathrm{A} \mathrm{Li}_{2} \mathrm{NiGe}_{3} \mathrm{O}_{8}$ pellet sintered at $1223 \mathrm{~K}$ for $24 \mathrm{~h}$ showed small additional Bragg peaks which are likely caused by lithium volatilisation, resulting in the onset of thermal decomposition and formation of impurities. Despite a rigorous search of the 2019 edition of the ICDD PDF-4+ database, we were unable to identify the additional phases present. It is noted that these peaks were not observed in data collected for a pellet sintered at the same temperature for $6 \mathrm{~h}$.

\subsection{Lithium-ion conductivity analysis by impedance spectroscopy}

Impedance data were collected for $\mathrm{Li}_{2} \mathrm{NiGe}_{3} \mathrm{O}_{8}$ pellets sintered for 6 and $24 \mathrm{~h}$. Owing to the lack of mixed valence $\mathrm{Ni}$ and $\mathrm{Ge}$ species in $\mathrm{Li}_{2} \mathrm{NiGe}_{3} \mathrm{O}_{8}$, the electrical conductivity is expected to be negligible, as previously reported. ${ }^{19,25}$ Hence, the responses observed in impedance data are likely due to $\mathrm{Li}^{+}$ion conduction. The complex impedance spectra obtained in Fig. 2(a) show two partly resolved arcs followed by a low frequency spike.

Irrespective of the sintering time, the arc observed at higher frequencies has an associated capacitance of $\sim 3.1 \times 10^{-12} \mathrm{~F}$, indicative of bulk, intra-granular responses. The second, intermediate frequency arc, with capacitance $\sim 1 \times 10^{-11} \mathrm{~F}$, is attributed to the response from the grain boundary component. ${ }^{29}$ Under both sintering conditions, a subsequent low-frequency Warburg spike, inclined at $\sim 50^{\circ}$ to the horizontal axis, was observed with associated capacitance of $\sim 10^{-6} \mathrm{~F}$, indicative of complete or partial ion blocking at the electrode, representative of ionic conductivity in $\mathrm{Li}_{2} \mathrm{NiGe}_{3} \mathrm{O}_{8}{ }^{29}$

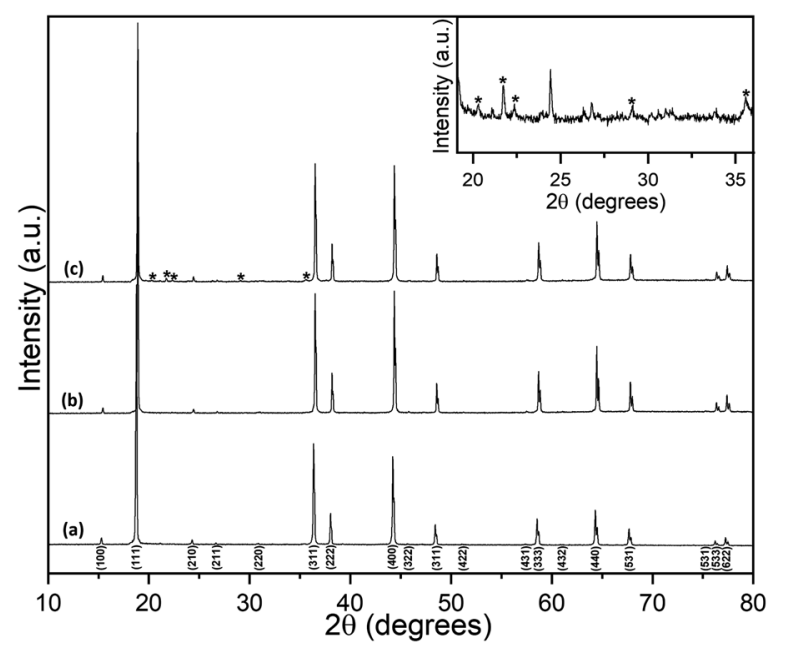

Fig. 1 XRD patterns obtained for (a) powdered $\mathrm{Li}_{2} \mathrm{NiGe}_{3} \mathrm{O}_{8}$ and pellets sintered for (b) $6 \mathrm{~h}$ and (c) $24 \mathrm{~h}$. Additional unknown reflections are denoted by *. An expansion of the $2 \theta$ range in (c) highlights the additional Bragg peaks present for the $24 \mathrm{~h}$ sintered pellet.
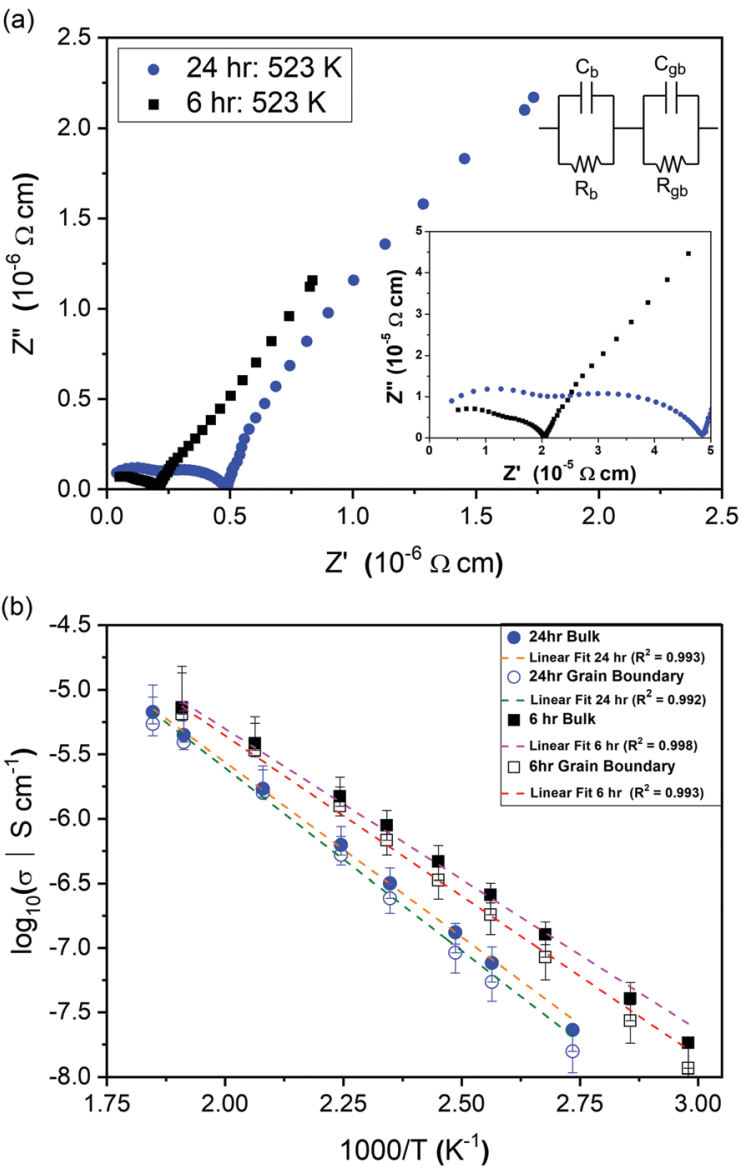

Fig. 2 (a) Complex impedance spectra collected at $\sim 523 \mathrm{~K}$ for $\mathrm{Li}_{2} \mathrm{NiGe}_{3} \mathrm{O}_{8}$ pellets prepared using different sintering times: 24 hours (blue circles) and 6 hours (black squares). (b) Arrhenius plots bulk and grain boundary conductivities for $\mathrm{Li}_{2} \mathrm{NiGe}_{3} \mathrm{O}_{8}$ pellets prepared using different sintering times: 24 hours (circles) and 6 hours (squares). Grain boundary activation energies of $0.56 \pm 0.02 \mathrm{eV}$ and $0.49 \pm 0.02 \mathrm{eV}$ were calculated for pellets sintered at 24 hours and 6 hours, respectively.

To extract conductivities from the arcs attributed to intragranular and grain boundary responses in the complex impedance spectra, fitting was performed using Z-View. Bulk conductivities were taken from the intersection of two manually fitted parallel RC semicircles, with errors calculated from the intersection of the semicircles with the $Z^{\prime}$ axis. The extracted bulk conductivities, as a function of inverse temperature, are shown in Fig. 2(b). For both samples, the data follows typical Arrhenius-type behaviour, enabling activation energies to be calculated. The pellet sintered for $6 \mathrm{~h}$ exhibited an activation energy of $0.46 \pm 0.01 \mathrm{eV}$, whilst the pellet fired for $24 \mathrm{~h}$ showed a higher value, $0.53 \pm 0.01 \mathrm{eV}$, closer to that previously reported by Kawai et al. $(0.55 \mathrm{eV})$ for $\mathrm{Li}_{2} \mathrm{NiGe}_{3} \mathrm{O}_{8}$ sintered for $24 \mathrm{~h}^{25}$ Assuming all $\mathrm{Li}$ in the spinel unit cell contribute to diffusion, an intra-grain diffusion coefficient, $D_{\mathrm{Li}}$, of $2.26 \times 10^{-13} \mathrm{~cm} \mathrm{~s}^{-1}$ can be estimated from impedance data at $336 \mathrm{~K}$ using the Nernst-Einstein equation: ${ }^{30}$

$$
D=\frac{\sigma k_{\mathrm{B}} T}{n_{\mathrm{Li}} e^{2}}
$$


It is evident from Fig. 2(a) and (b) that longer sintering times leads to a concomitant decrease in both bulk and grain boundary conductivities. This difference is attributed to the loss of lithium and subsequent formation of additional phases during sintering at temperatures close to the reported melting point of $\mathrm{Li}_{2} \mathrm{NiGe}_{3} \mathrm{O}_{8}$ $(1243 \mathrm{~K}) .^{25}$

\subsection{Probing Li diffusion using solid-state NMR}

Using solid-state NMR spectroscopy it is possible to probe both local structural changes and ion mobility. Both lithium nuclides $\left({ }^{6} \mathrm{Li}\right.$ and $\left.{ }^{7} \mathrm{Li}\right)$ are suitable for NMR spectroscopy, however, one is often preferred over the other, depending on the specific system under investigation. Typically, ${ }^{7} \mathrm{Li}$ is favoured because of its higher natural abundance (92.5\%) when compared to ${ }^{6} \mathrm{Li}(7.5 \%)$. However, its large quadrupole moment can give rise to relatively broad lines when $\mathrm{Li}$ is in a low symmetry site. In such cases, ${ }^{6} \mathrm{Li}$ is often preferred because it can allow close or overlapped resonances to be resolved. Moreover, because of its smaller quadrupolar interaction, the ${ }^{6} \mathrm{Li}$ shift may be more closely approximate to the isotropic chemical shift, thereby providing a better measure of the Li bonding environment. ${ }^{31}$ The ${ }^{7} \mathrm{Li}$ and ${ }^{6} \mathrm{Li}$ MAS NMR spectra obtained for $\mathrm{Li}_{2} \mathrm{NiGe}_{3} \mathrm{O}_{8}$ are shown in Fig. 3(a) and (b), respectively. The ${ }^{7}$ Li MAS NMR spectrum acquired at $10 \mathrm{kHz}$ exhibits a lineshape characteristic of a

(a)

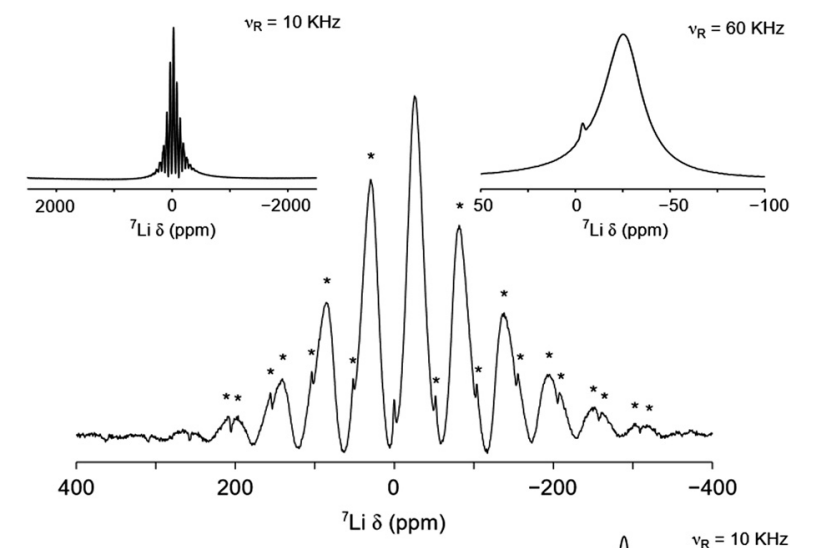

(b)

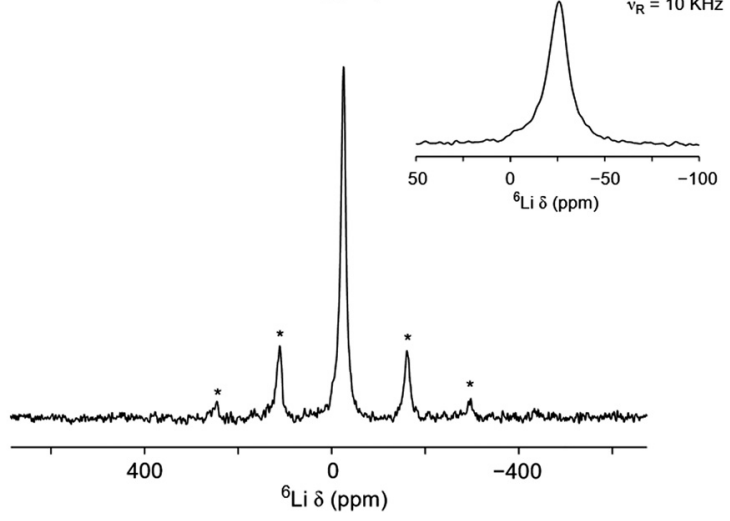

Fig. 3 (a) ${ }^{7} \mathrm{Li}$ and (b) ${ }^{6} \mathrm{Li}$ MAS NMR spectra acquired for $\mathrm{Li}_{2} \mathrm{NiGe}_{3} \mathrm{O}_{8}$. In (a), two different MAS rates were used, 10 and $60 \mathrm{kHz}$, respectively. In (b), a MAS rate of $10 \mathrm{kHz}$ was used and an expansion of the central transition is also shown as a comparison. All spinning sidebands are denoted by *. paramagnetic-containing species, i.e., a complex manifold of spinning sidebands. The spectrum obtained indicates the presence of two resonances at $\delta \approx-25$ and $0 \mathrm{ppm}$, likely corresponding to two distinct $\mathrm{Li}$ sites, in good agreement with the reported $\mathrm{P}_{3} 32$ phase of $\mathrm{Li}_{2} \mathrm{NiGe}_{3} \mathrm{O}_{8}$.

The variation in ${ }^{6,7} \mathrm{Li}$ chemical shift with coordination number is well reported for a variety of crystalline systems. ${ }^{32}$ Typically, in diamagnetic systems, $\mathrm{LiO}_{4}$ environments exhibit chemical shifts between $\delta_{\text {iso }}=0-3$ ppm, whilst $\mathrm{LiO}_{6}$ environments are between -1 and $0 \mathrm{ppm} .^{31,32}$ In paramagnetic systems, these shifts can be altered substantially by the presence of a paramagnetic species, i.e., the presence of an unpaired electron(s). ${ }^{33,34}$

Based on the chemical shifts reported in the literature, coupled with the X-ray diffraction data presented here, and the relative intensities of each resonance, the resonance at $\delta \approx-25 \mathrm{ppm}$ is believed to correspond to the tetrahedral $8 c$ site and the resonance at $\delta \approx 0.0 \mathrm{ppm}$ corresponds to the octahedral $12 d$ site. Hence, at room temperature, it appears that small amounts of Li occupy the vacant octahedral site, which is in contrast to previous diffraction studies. ${ }^{26}$ In the spectrum, the tetrahedral site is shifted from its expected position, indicating that it is directly affected by the presence of paramagnetic $\mathrm{Ni}^{2+}$, i.e., it is experiencing a Knight shift, although the shift observed here is relatively small when compared to similar reported systems where the resonance can be shifted by several hundreds of ppm. ${ }^{33,34} \mathrm{~A}$ fast spinning $(60 \mathrm{kHz}){ }^{7} \mathrm{Li}$ MAS NMR spectrum was also acquired for $\mathrm{Li}_{2} \mathrm{NiGe}_{3} \mathrm{O}_{8}$ (shown as an inset in Fig. 3(a)), which revealed the presence of two resonances, one broad resonance at $\delta=-25.3 \mathrm{ppm}$, belonging to the tetrahedral $\mathrm{Li}$ site and a sharper resonance at $\delta=-3.9 \mathrm{ppm}$ corresponding to the octahedral $\mathrm{Li}$ site. It is noted that the extra resolution afforded by faster MAS rates indicates that a Knight shift is also observed for the octahedral site. At slower MAS rates the lineshape is broadened, which hinders the extraction of an accurate shift for this site. Again, the intensities indicate that a small quantity of $\mathrm{Li}$ is occupying the octahedral sites. To verify the quantity of $\mathrm{Li}$ on each site, the spectrum was fitted to determine the approximate ratio of tetrahedral to octahedral sites. It is noted that this is somewhat challenging based on the broadened nature and overlap of the two sites. Occupancies of 0.024 and 0.976 were obtained for the octahedral and tetrahedral sites, respectively (Fig. S4, ESI $\dagger$ ). This is in good agreement with the slow spinning $(10 \mathrm{kHz}){ }^{7} \mathrm{Li}$ MAS NMR spectrum.

Initial variable-temperature ${ }^{6} \mathrm{Li}$ MAS NMR studies of $\mathrm{Li}_{2} \mathrm{Ni}$ $\mathrm{Ge}_{3} \mathrm{O}_{8}$ were completed over the temperature range 260 to $412 \mathrm{~K}$. The corresponding spectra are shown in Fig. 4(a). Between 260 and $357 \mathrm{~K}$, a single broad resonance is observed. As the temperature is increased to $379 \mathrm{~K}$ a second resonance appears at $\delta=-1.1 \mathrm{ppm}$, indicating the presence of a second Li site, believed to correspond to the octahedral Li site. The appearance of this site upon heating indicates diffusion of the Li ions from the tetrahedral $8 c$ site onto one of the three neighbouring vacant $12 d$ octahedral sites via a hopping mechanism. The presence of a second site is in good agreement with previous high temperature $\left(\sim 350-850{ }^{\circ} \mathrm{C}\right)$ ToF ND structural studies of $\mathrm{Li}_{2} \mathrm{NiGe}_{3} \mathrm{O}_{8}{ }^{26}{ }^{26}$ However, the solid-state NMR data presented 


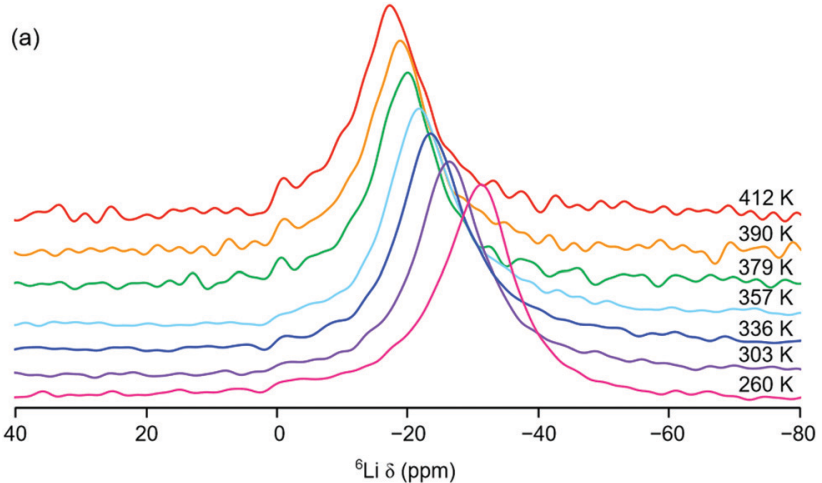

(b)

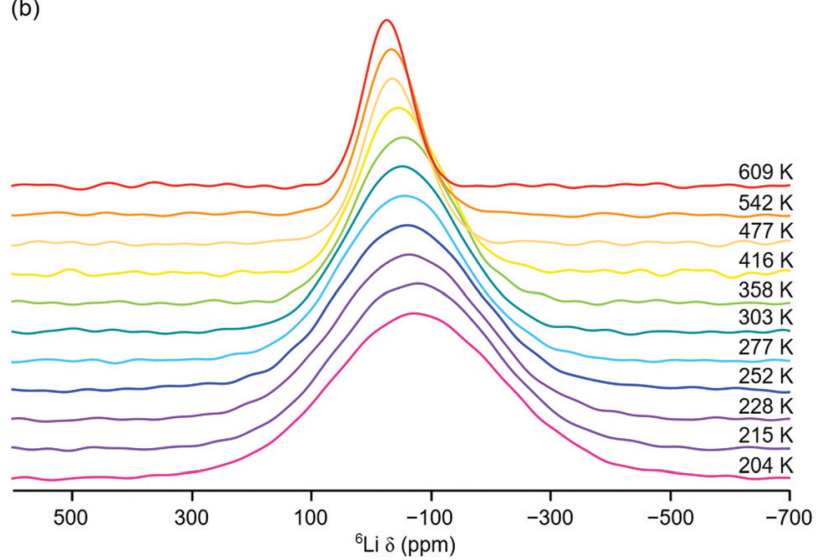

Fig. 4 Variable-temperature ${ }^{6} \mathrm{Li}$ (a) MAS and (b) static NMR spectra obtained for $\mathrm{Li}_{2} \mathrm{NiGe}_{3} \mathrm{O}_{8}$. The spectra in (a) were acquired over the temperature range 260 and $412 \mathrm{~K}$ and a MAS rate of $10 \mathrm{kHz}$ was used. All of the spectra shown in (b) were acquired under static conditions over the temperature range 204 to $609 \mathrm{~K}$.

indicate that Li diffusion onto the octahedral site(s) occurs at lower temperatures than previously reported. ${ }^{26}$ In fact, our ${ }^{7}$ Li MAS NMR data (vide supra) suggest that, even at room temperature, some of the vacant octahedral $12 d$ sites are occupied. However, owing to relatively poor signal-to-noise in the VT ${ }^{6} \mathrm{Li}$ MAS NMR spectra, it is challenging to accurately determine the precise temperature at which $\mathrm{Li}$ ions become mobile and move between the $8 c$ and $12 d$ sites.

As the temperature is increased, there is an obvious, yet gradual, linear change in chemical shift of the resonance corresponding to the tetrahedral site, towards more positive values. For example, the resonance is centred at $\delta=-31.3 \mathrm{ppm}$ at $260 \mathrm{~K}$ and moves to $\delta=-17.2 \mathrm{ppm}$ at $412 \mathrm{~K}$. This is in contrast to the trend expected with increasing temperature for a paramagnetic system.

Typically, as temperature is increased, Li chemical shift values move to lower (or more negative) values, thereby obeying Curie-Weiss behaviour. ${ }^{35}$ As stated earlier, a Knight shift is observed for the tetrahedral site in the ${ }^{7}$ Li MAS NMR spectrum obtained at room temperature. This indicates that the tetrahedral Li site is closer in the structure to the paramagnetic $\mathrm{Ni}^{2+}$ centre. In contrast, the resonance assigned to the octahedral $12 d$ site exhibits a very small change in chemical shift, moving from $\delta=-1.1 \mathrm{ppm}$ to $\delta=-0.9 \mathrm{ppm}$, with increasing temperature.
It is noted that, owing to the relatively low intensity and broad nature of the resonance, it is challenging to accurately determine the precise chemical shift observed. However, the resonance is not shifted to the same extent as that corresponding to the tetrahedral Li site. This suggests that the octahedral $12 d$ site is positioned further away from the $\mathrm{Ni}^{2+}$ in the structure and therefore does not experience the same effect of the paramagnetic species, i.e., no significant Knight shift. As temperature is increased and Li ions increasingly migrate from the tetrahedral site onto the octahedral site they move further and further away from the paramagnetic species. As a result, their chemical shift changes (becoming more positive). $\mathrm{In}_{2} \mathrm{NiGe}_{3} \mathrm{O}_{8}$, the octahedral Li site is $\sim 4 \AA$ from the Ni centre, compared to a separation of $\sim 3.2 \AA$ between the tetrahedral site to the Ni centre. Similar VT ${ }^{7}$ Li MAS NMR experiments were completed between 260 and $357 \mathrm{~K}$ and the same trend in chemical shift was observed, Fig. S5 in the ESI. $\dagger$ Hence, the VT ${ }^{6} \mathrm{Li}$ and ${ }^{7} \mathrm{Li}$ MAS NMR data are in good agreement.

To further investigate $\mathrm{Li}^{+}$ion mobility in $\mathrm{Li}_{2} \mathrm{NiGe}_{3} \mathrm{O}_{8}$, additional static variable-temperature ${ }^{6} \mathrm{Li}$ NMR experiments were completed over a greater temperature range (204 to $609 \mathrm{~K}$ ). It is noted that, due to hardware limitations, it is not possible to acquire ${ }^{6} \mathrm{Li}$ MAS NMR data over this temperature range. The VT static ${ }^{6} \mathrm{Li}$ NMR spectra obtained are shown in Fig. 4(b). Below room temperature, a single broad and relatively featureless lineshape is observed. As the temperature is increased, line narrowing is observed, indicative of $\mathrm{Li}^{+}$ion mobility within $\mathrm{Li}_{2} \mathrm{NiGe}_{3} \mathrm{O}_{8}$, in good agreement with the VT ${ }^{6} \mathrm{Li}$ MAS NMR data.

The variation in ${ }^{6} \mathrm{Li}$ chemical shift and full width at half maximum (FWHM) with increasing temperature are shown in Fig. 5, where a linear change in chemical shift is observed and a gradual narrowing of the resonance is also observed, again indicative of $\mathrm{Li}$ ion motion. In contrast to the MAS NMR data, it was not possible to resolve distinct $\mathrm{Li}$ sites or the individual contributions from the $8 c$ tetrahedral and $12 d$ octahedral $\mathrm{Li}$ sites in the observed lineshape. In this instance, the ${ }^{6} \mathrm{Li} \mathrm{NMR}$ data is only capable of identifying the presence of $\mathrm{Li}^{+}$ion mobility within $\mathrm{Li}_{2} \mathrm{NiGe}_{3} \mathrm{O}_{8}$ and not the precise contribution of each site. To gain further insight into the ion mobility, ${ }^{6} \mathrm{Li} T_{1}$ measurements were attempted for $\mathrm{Li}_{2} \mathrm{NiGe}_{3} \mathrm{O}_{8}$ using a saturation recovery experiment. However, owing to the paramagnetic nature of the sample, the relaxation properties of the system were too fast and could not be accurately measured. Similarly, using the VT NMR data acquired, and analysis of the corresponding FWHM, attempts were made to obtain an activation energy for Li migration. Unfortunately, due to a combination of hardware limitations and the specific characteristics of the system under investigation, we were unable to acquire the full motional narrowing curve for $\mathrm{Li}_{2} \mathrm{NiGe}_{3} \mathrm{O}_{8}$ (Fig. 5(b)). Hence, we were unable to obtain an activation energy.

\subsection{Investigation of intra-grain diffusion by $\mu \mathrm{SR}$}

To explore $\mathrm{Li}^{+}$ion diffusion in greater depth, muon spectroscopy, $\mu \mathrm{SR}$, was used. Positively charged muons, $\mu^{+}$, have a lifetime of $2.197 \mu$ s and are $100 \%$ spin polarised. They can be implanted $\sim 200 \mu \mathrm{m}$ into a sample and are expected to reside 

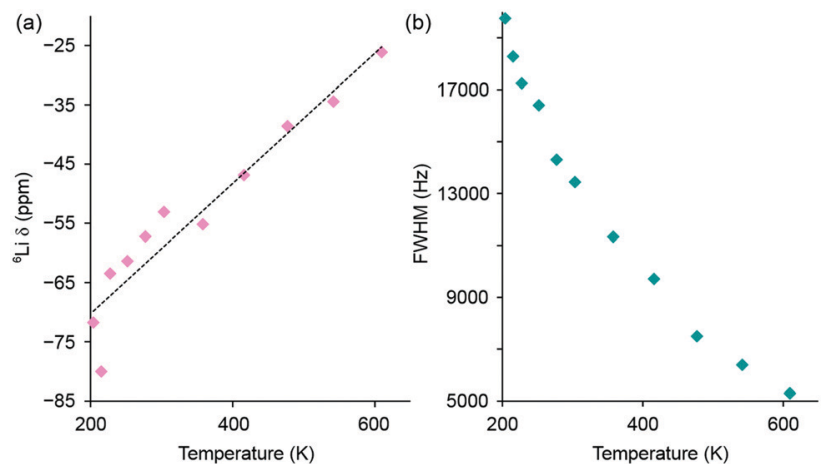

Fig. 5 Variation in (a) ${ }^{6} \mathrm{Li}$ chemical shift and (b) full width at half maximum (FWHM) with increasing temperature for the ${ }^{6} \mathrm{Li}$ static NMR data obtained for $\mathrm{Li}_{2} \mathrm{NiGe}_{3} \mathrm{O}_{8}$. The corresponding spectra are shown in Fig. 4(b).

near oxygen sites. ${ }^{36-39}$ The $\mu^{+}$spin can precess depending on the different magnetic fields it experiences, such as external applied fields, fields from nearby nuclei, or those from unpaired electrons in paramagnetic species. The diffusion of $\mathrm{Li}^{+}$ions leads the $\mu^{+}$to undergo a spin flip due to the inherent nuclear magnetic moment of lithium. When the $\mu^{+}$decays, the positron produced is most likely to be emitted in the direction of the $\mu^{+}$spin at decay. By recording the positrons emitted from opposite sides of the sample, the asymmetry of positrons can then be followed as a function of time and the evolution of the muon spin ensemble deduced.

Muon decay asymmetry data collected in longitudinal magnetic fields of 0,5 and $10 \mathrm{G}$ at various temperatures were fitted using WiMDA. For each temperature point, data from all three fields were fitted to a single model using Keren's analytic generalisation of the Abragam function, multiplied by an exponential relaxation term to account for the presence of paramagnetic Ni. ${ }^{40}$ Example fitted data for $\mathrm{Li}_{2} \mathrm{NiGe}_{3} \mathrm{O}_{8}$ at room temperature are shown in Fig. 6 .

From the different fits, the calculated fluctuation rate, $\nu$, and local magnetic field distribution, $\Delta$, were extracted and their

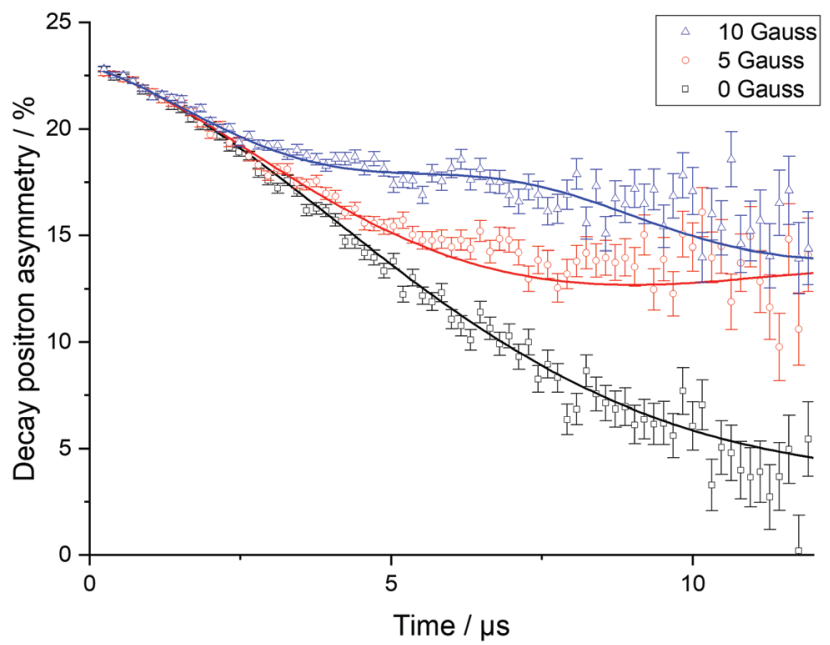

Fig. 6 Raw muon spectroscopy data for $\mathrm{Li}_{2} \mathrm{NiGe}_{3} \mathrm{O}_{8}$, collected at $300 \mathrm{~K}$ in a zero field (squares), and applied longitudinal fields of $5 \mathrm{G}$ (circles) and $10 \mathrm{G}$ (triangles), with fits using the Keren function with a Lorentzian relaxation.
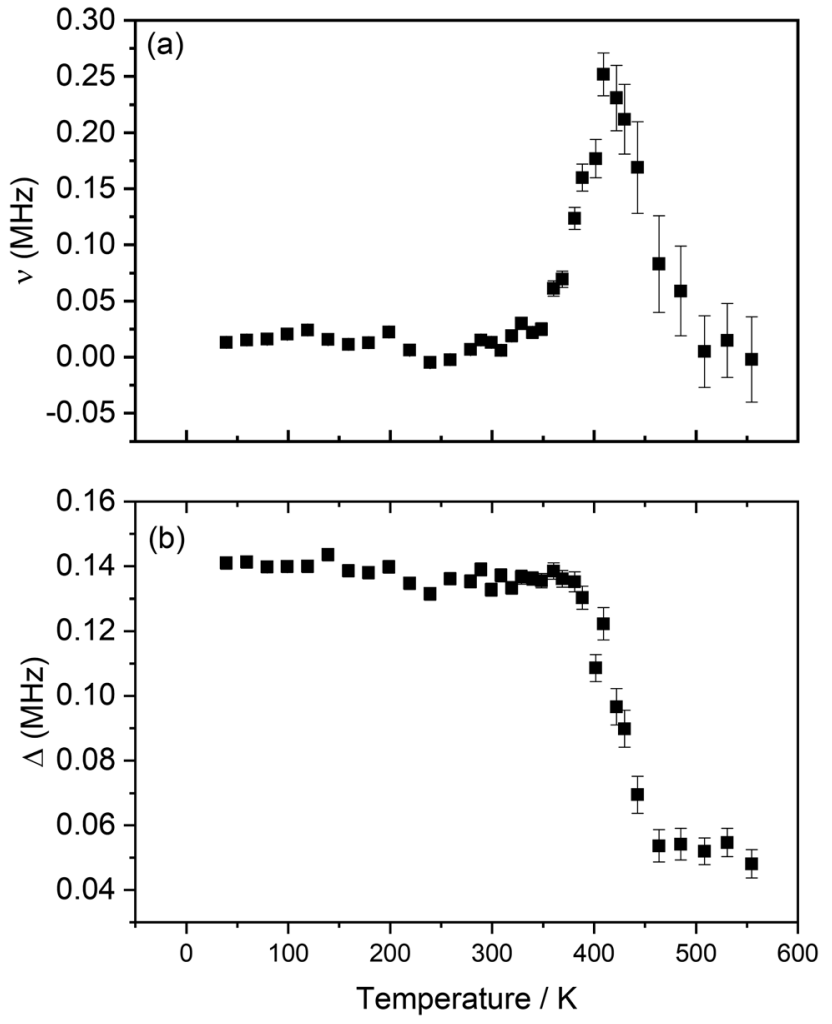

Fig. 7 Variation of $\nu$ (a) and $\Delta$ (b) with temperature obtained from the fitting of raw asymmetry data to the Keren function for $\mathrm{Li}_{2} \mathrm{NiG}_{3} \mathrm{O}_{8}$, measured from $40 \mathrm{~K}$ to $555 \mathrm{~K}$.

variation with temperature is shown in Fig. 7(a) and (b). The fluctuation rate shows a plateau at lower temperatures, followed by an Arrhenius-like increase due to thermally activated diffusion of lithium ions above $\sim 300 \mathrm{~K}$. This increase in fluctuation rate continues to $421 \mathrm{~K}$; the subsequent decrease observed at higher temperatures is likely indicative of $\mathrm{Li}^{+}$ions hopping at rates too fast for $\mu$ SR to capture. ${ }^{36,37}$ The values obtained for $\Delta$, Fig. 7(b), support this hypothesis; $\Delta$ is constant, within errors, at low temperatures, due to the slow rate of lithium hopping, but then shows a significant decrease from $\sim 380 \mathrm{~K}$ related to the effect of motional narrowing, where $\mathrm{Li}^{+}$ions are moving quickly and their nuclear spins differ from point to point within the sample. Above $442 \mathrm{~K}$, lithium diffusion coefficients become too large for the muons to capture, and $\Delta$ values plateau again.

\subsection{Elucidating diffusion mechanisms}

The lithium ion diffusion coefficient for $\mathrm{Li}_{2} \mathrm{NiGe}_{3} \mathrm{O}_{8}$ can be calculated using the equation:

$$
D_{\mathrm{Li}}=\sum_{i=1}^{n} \frac{1}{N_{i}} Z_{\nu, i} S_{i}^{2} \nu
$$

where $N_{i}$ is the number of accessible Li sites in the $i$ th path, $Z_{\nu, i}$ is the vacancy fraction of the destination sites in the $i$ th path, $S_{i}$ is the hopping distance between sites, and $\nu$ is the fluctuation rate at $300 \mathrm{~K}$, and assumed to be related to $\mathrm{Li}^{+}$ions hopping between $8 c$ and $12 d$ crystallographic sites, as reported previously. ${ }^{26,41}$ 
Based on the findings obtained using $\mu \mathrm{SR}$ and solid-state NMR spectroscopy, additional analysis was conducted on the crystal structural refinements, using the ToF ND data previously reported by our group. ${ }^{26}$ Therein, refinements conducted on room temperature data had indicated within standard statistical approaches that the $8 c$ site was fully and solely occupied by $\mathrm{Li}$; occupancy of the $12 d$ migratory interstitial had only been noted where datasets had been collected at temperatures above ambient conditions. This does not preclude the possibility of very low concentrations of lithium hopping between the two sites at, or close to, ambient conditions - such results would merely be statistically indistinguishable from the fully ordered structure reported. This assumption is in good agreement with the ${ }^{6,7} \mathrm{Li}$ MAS NMR and $\mu$ SR data presented (Fig. 3 and 7). Therefore, in order to calculate the true diffusion coefficient at $300 \mathrm{~K}$, refined occupancies of both the $8 \mathrm{c}$ and $12 d$ sites from $350 \leq T /{ }^{\circ} \mathrm{C} \leq 850$ were linearly extrapolated (Fig. S6, ESI $\dagger$ ) to give proposed fractional occupancies, or $Z_{\nu, i}$, of 0.97 and 0.03 for the $8 \mathrm{c}$ and $12 d$ sites respectively at $300 \mathrm{~K}$ (Table S2, ESI $\dagger$ ). This is in good agreement with the occupancies obtained from the ${ }^{7} \mathrm{Li}$ MAS NMR data (vide supra).

The hopping distance, $S_{i}$, in $\mathrm{Li}_{2} \mathrm{NiGe}_{3} \mathrm{O}_{8}$ can be calculated by considering that an ion hopping between the $8 c$ and $12 d$ sites likely passes through three saddle points (SP) along the way, in the $\mathrm{LiO}_{4}$ tetrahedral and $\mathrm{LiO}_{6}$ octahedral faces, and an empty tetrahedral site. Once in the $12 d$ site, $\mathrm{Li}^{+}$ions can hop to one of two neighbouring $8 c$ sites (one of which is the original $8 c$ site). A reasonable hopping distance of $2.95 \AA$ can thus be calculated from the distances between the $8 c$-SP1-SP2-SP3-12 $d$ sites along this proposed conduction pathway (Fig. 8).

By following these assumptions, the calculated diffusion coefficient, $D_{\mathrm{Li}}$, for $\mathrm{Li}_{2} \mathrm{NiGe}_{3} \mathrm{O}_{8}$ at $300 \mathrm{~K}$ is found to be $3.89 \times$ $10^{-12} \mathrm{~cm}^{2} \mathrm{~s}^{-1}$ (Table S2, ESI $\dagger$ ). This is in good agreement with the figure extracted from impedance spectroscopy data, and is similar to values reported for other spinel-type materials, e.g., $\mathrm{Li}_{4} \mathrm{Ti}_{5} \mathrm{O}_{12}$ which has a diffusion coefficient, $D_{\mathrm{Li}}$ of between $3.2 \times$

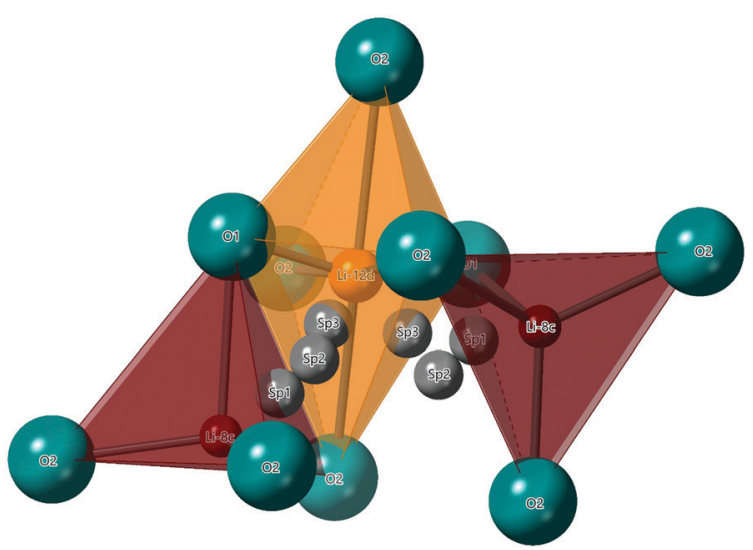

Fig. 8 Portion of $\mathrm{Li}_{2} \mathrm{NiGe}_{3} \mathrm{O}_{8}$ crystal structure showing the proposed $8 c-S P 1-S P 2-S P 3-12 d$ conduction pathway. A $\mathrm{Li}_{12 d}-\mathrm{O}_{6}$ octahedron is shown in orange and the two nearest $\mathrm{Li}_{8 c}-\mathrm{O}_{4}$ tetrahedral are shown in red. A hopping distance of $2.95 \AA$ can be calculated from a $\mathrm{Li}_{8 \mathrm{c}}$-ion hopping between three possible saddle points (grey) into the neighbouring $\mathrm{Li}_{12 d}$ site.

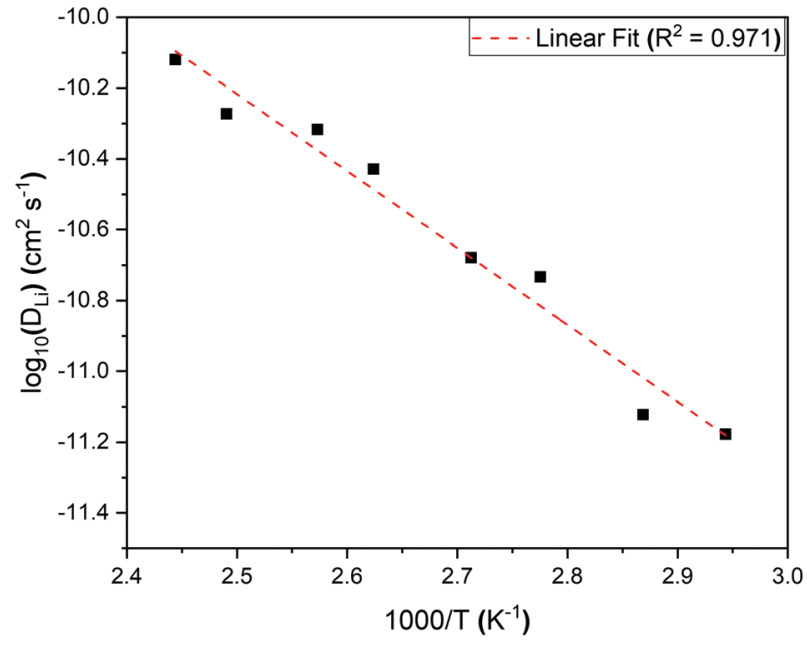

Fig. 9 Arrhenius plot of the diffusion coefficient in the temperature range $340 \mathrm{~K}$ to $410 \mathrm{~K}$ for the complex spinel, $\mathrm{Li}_{2} \mathrm{NiGe}_{3} \mathrm{O}_{8}$. The calculated activation energy from the slope is $0.43 \pm 0.03 \mathrm{eV}$.

$10^{-11}$ to $4.0 \times 10^{-12} \mathrm{~cm}^{2} \mathrm{~s}^{-1}$, depending on the technique used and precise degree of lithiation. ${ }^{42,43}$ By comparison, other solid electrolyte candidate materials based on the Li-stuffed garnet structure, e.g., $\mathrm{Li}_{6.5} \mathrm{Al}_{0.25} \mathrm{La}_{2.92} \mathrm{Zr}_{2} \mathrm{O}_{12}$, with a faster ionic conductivity and shorter hopping distance $(1.67 \AA)$, has $\mathrm{a} \mathrm{Li}^{+}$ion diffusion coefficient of $4.62 \times 10^{-11} \mathrm{~cm}^{2} \mathrm{~s}^{-1} \cdot 39$

An activation energy of $0.43 \pm 0.03 \mathrm{eV}$ for lithium hopping in our powdered sample of $\mathrm{Li}_{2} \mathrm{NiGe}_{3} \mathrm{O}_{8}$ was calculated from an Arrhenius plot of $\log D_{\mathrm{Li}}$ over the thermally activated region (Fig. 9), in good agreement with the value obtained from EIS data on a pellet sintered for $6 \mathrm{~h}(0.46 \pm 0.01 \mathrm{eV})$, but considerably lower than that observed after a $24 \mathrm{~h}$ sinter $(0.53 \pm$ $0.01 \mathrm{eV}$ ). This is further evidence that long dwell times at temperatures close to the melting point result in $\mathrm{Li}$ loss through volatilisation. As a result, additional studies aimed at identifying less extreme consolidation methods are currently underway. Length-scale dependent ion dynamics are well known in the literature with $\mathrm{LiFePO}_{4}$ being a particular prominent example, ${ }^{44}$ where the activation energy changes several-fold and the diffusion constant by several orders-of-magnitude. $\mathrm{In}_{\mathrm{Li}} \mathrm{NiGe}_{3} \mathrm{O}_{8}$ the change in activation energy is far less pronounced.

\section{Conclusions}

Lithium-ion diffusion mechanisms in the complex spinel $\mathrm{Li}_{2} \mathrm{Ni}$ $\mathrm{Ge}_{3} \mathrm{O}_{8}$ have been investigated using a range of complementary techniques. Building on previous reports of an $8 c-12 d-8 c$ conduction pathway, using $\mu \mathrm{SR}$ and ${ }^{6,7} \mathrm{Li}$ solid-state NMR spectroscopy, we have shown that $\mathrm{Li}^{+}$ion hopping becomes thermally activated at $\sim 300 \mathrm{~K}$, resulting in partial occupancy of the migratory interstitial site at lower temperatures than previously reported. A lithium diffusion coefficient of $3.89 \times 10^{-12} \mathrm{~cm}^{2} \mathrm{~s}^{-1}$ at $300 \mathrm{~K}$ and an activation energy for lithium hopping of $0.43 \pm 0.03 \mathrm{eV}$ were determined using $\mu \mathrm{SR}$ on powdered samples. This energy is considerably lower than that observed for sintered pellets $(0.46 \pm 0.01$ and $0.53 \pm 0.01 \mathrm{eV}$, 
depending on the sintering conditions), suggesting that the investigation of an alternate consolidation route, avoiding $\mathrm{Li}$ volatilisation, may be worthwhile. This work also highlights the necessity of using multiple complementary techniques, such as impedance spectroscopy, solid-state NMR and $\mu \mathrm{SR}$, as local probes in order to gain a more complete understanding of hopping mechanics in important ionic conductors.

\section{Conflicts of interest}

There are no conflicts to declare.

\section{Acknowledgements}

The PhD work of DZCM under the supervision of NRM and RB was funded by EPSRC through a Doctoral Training Partnership grant awarded to the University of Sheffield. RB acknowledges support by the Lloyd's Register Foundation and Royal Academy of Engineering under the Research Fellowships scheme. The authors extend their gratitude to the ISIS Neutron \& Muon Facility for the award of EMU Beamtime. KEJ and ARH would like to thank the EPSRC Doctoral Training Partnership EP/N509462/1 for the award of a studentship to ARH and Dr David Apperley for experimental assistance.

\section{References}

1 M. Armand and J.-M. Tarascon, Nature, 2008, 451, 652-657.

2 J. B. Goodenough and K. Park, J. Am. Chem. Soc., 2013, 135, 1167-1176.

3 N. Nitta, F. Wu, J. T. Lee and G. Yushin, Mater. Today, 2015, 18, 252-264.

4 P. G. Balakrishnan, R. Ramesh and T. P. Kumar, J. Phys. Chem. Lett., 2006, 155, 401-414.

5 J. B. Goodenough and Y. Kim, Chem. Mater., 2010, 22, 587-603.

6 Q. Li, J. Chen, L. Fan, X. Kong and Y. Lu, Green Energy Environ., 2016, 1, 18-42.

7 Samsung, 2016, http:/www.samsung.com/us/note7recall/.

8 J. C. Bachman, S. Muy, A. Grimaud, H. Chang, N. Pour, S. F. Lux, O. Paschos, F. Maglia, S. Lupart, P. Lamp and L. Giordano, Chem. Rev., 2016, 116, 140-162.

9 N. Kamaya, K. Homma, Y. Yamakawa, M. Hirayama, R. Kanno, M. Yonemura, T. Kamiyama, Y. Kato, S. Hama and K. Kawamoto, Nat. Mater., 2011, 10, 682-686.

10 Y. Li, J.-T. Han, C.-A. Wang and J. B. Goodenough, J. Mater. Chem., 2012, 3, 15357-15361.

11 R. Kanno and M. Murayama, J. Electrochem. Soc., 2001, 148, 742-746.

12 A. C. Luntz, J. Voss and K. Reuter, J. Phys. Chem. Lett., 2015, 6, 4599-4604.

13 J. Kasemchainan, P. G. Bruce and J. Matthey, Technol. Rev., 2018, 62, 177-180.

14 V. Thangadurai, S. Narayanan and D. Pinzaru, Chem. Rev., 2014, 43, 4714-4727.

15 A. Orera and J. Campo, Inorg. Chem., 2016, 55, 1324-1332.
16 K. Hofstetter, A. J. Samson, S. Narayanan and V. Thangadurai, J. Power Sources, 2018, 390, 297-312.

17 W. H. Bragg, Philos. Mag., 1915, 30, 305-315.

18 S. Nishikawa, Proc. Math. Phys. Soc. Tokyo, 1915, 8, 199-209.

19 M. Nakayama, R. Jalem and T. Kasuga, Solid State Ionics, 2014, 262, 74-76.

20 H. Kawai, M. Nagata, H. Tukamoto and A. R. West, Electrochem. Solid-State Lett., 1998, 1, 212-214.

21 A. Manthiram, K. Chemelewski and E. Lee, Energy Environ. Sci., 2014, 7, 1339-1350.

22 M. M. Thackeray, J. Am. Ceram. Soc., 1999, 54, 3347-3354.

23 B. M. Wagemaker, D. R. Simon, E. M. Kelder, J. Schoonman, C. Ringpfeil, U. Haake, D. Lützenkirchen-hecht, R. Frahm and F. M. Mulder, Adv. Mater., 2006, 18, 3169-3173.

24 T. Ohzuku, A. Ueda and N. Yamamota, J. Electrochem. Soc., 1995, 142, 1431-1435.

25 H. Kawai, M. Tabuchi, M. Nagata, H. Tukamoto and A. R. West, J. Mater. Chem., 1998, 8, 1273-1280.

26 N. Reeves-Mclaren, R. I. Smith and A. R. West, Chem. Mater., 2011, 23, 3556-3563.

27 B. H. Toby and R. B. Von Dreele, J. Appl. Crystallogr., 2013, 46, 544-549.

28 F. L. Pratt, Phys. B, 2000, 289-290, 710-714.

29 J. T. S. Irvine, D. C. Sinclair and A. R. West, Adv. Mater., 1990, 2, 132-138.

30 M. M. Ahmad, RSC Adv., 2015, 25824-25829.

31 K. MacKenzie and S. Mark, Multinuclear Solid-State Nuclear Magnetic Resonance of Inorganic Materials (Pergamon Materials Series), Elsevier Science Ltd, 2002, pp. 629-639.

32 Z. Xu and J. F. Stebbins, Solid State Nucl. Magn. Reson., 1995, 5, 103-112.

33 Y. J. Lee, F. Wang and C. P. Grey, J. Am. Chem. Soc., 1998, 120, 12601-12613.

34 C. P. Grey and Y. J. Lee, Solid State Sci., 2003, 5, 883-894.

35 L. S. Cahill, R. P. Chapman, C. W. Kirby and G. R. Goward, Appl. Magn. Reson., 2007, 32, 565-581.

36 P. J. Baker, I. Franke, F. L. Pratt, T. Lancaster, D. Prabhakaran, W. Hayes and S. J. Blundell, Phys. Rev. B: Condens. Matter Mater. Phys., 2011, 84, 1-8.

37 M. Mansson and J. Sugiyama, Phys. Scr., 2013, 88, 1-13.

38 M. J. Ariza, D. J. Jones, J. Roziere, J. S. Lord and D. Ravot, J. Phys. Chem. B, 2003, 107, 6003-6011.

39 M. Amores, T. E. Ashton, P. J. Baker, E. J. Cussen and S. A. Corr, J. Mater. Chem. A, 2016, 4, 1729-1736.

40 A. Keren, Phys. Rev. B: Condens. Matter Mater. Phys., 1994, 50, 10039-10042.

41 R. J. Borg and G. J. Dienes, An Introduction to Solid State Diffusion, Academic, San Diego, CA, 1988.

42 J. Sugiyama, H. Nozaki, I. Umegaki, K. Mukai, K. Miwa, S. Shiraki, T. Hitosugi, A. Suter, T. Prokscha, Z. Salman, J. S. Lord and M. Martin, Phys. Rev. B: Condens. Matter Mater. Phys., 2015, 92, 1-9.

43 W. J. H. Borghols, M. Wagemaker, U. Lafont and E. M. Kelder, J. Phys. Chem. B, 2009, 12, 17786-17792.

44 G. Krishna, P. Dathar, D. Sheppard, K. J. Stevenson and G. Henkelman, Chem. Mater., 2011, 4032-4037. 